\title{
Avaliação de programas educacionais no contexto da educação remota e covid 19
}

\section{Evaluation of Educational Programs in the Context of Remote Education and COVID 19}

Francisco José Candido dos Reis ${ }^{1}$ (D), Anderson Marliere Navarro² (D)

\begin{abstract}
RESUMO
No início de 2020 o mundo foi surpreendido pelo surgimento e rápido avanço da Covid-19. O impacto sobre os programas educacionais foi enorme. Sendo o distanciamento social uma das poucas formas de mitigar o avanço da pandemia, o ensino remoto emergencial (ERE) passou a ser uma imposição. Neste contexto ganha grande relevância avaliar estas iniciativas a fim de promover rapidamente as mudanças necessárias para que se consiga, minimamente, cumprir os objetivos dos programas educacionais.
\end{abstract}

Neste artigo discutiremos os fundamentos para a avaliação de programas educacionais no contexto da ERE e da Covid-19. Apresentaremos os princípios básicos de uma avaliação de programas educacionais que são aplicáveis desde a uma aula isoladamente, até ao curso como um todo. Discutiremos a aplicação dos modelos experimentais, Kirkpatrick, modelo lógico e CIPP (Contexto, Insumo, Processo e Produto).

A avaliação das atividades educacionais que utilizaram o ERE, implementado durante a pandemia em 2020, é uma oportunidade para rever práticas e induzir mudanças substanciais no processo de formação das próximas gerações de profissionais da saúde e dos docentes.

Palavras-chave: Avaliação de programas educacionais; Educação a distância; Covid-19

\begin{abstract}
:
In early 2020 the world was surprised by the emergence and rapid advance of Covid-19. The impact on educational programs was enormous. Since social distancing is one of the few ways to mitigate the pandemic's advance, emergency remote education (ERE) has become an imposition. In this context, it is of great relevance to evaluate these initiatives to quickly promote the necessary changes so that it is possible, at least, to fulfill the objectives of the educational programs.
\end{abstract}

In this article, we discuss the fundamentals for evaluating educational programs in the context of ERE and Covid-19. We present the basic principles of evaluation applicable from a single class to a course as a whole. We discuss the application of the following models: experimental, Kirkpatrick, logical model, and CIPP (Context, Input, Process, and Product).

The evaluation of the educational activities that used the ERE, implemented during the pandemic in 2020, is an opportunity to review practices and induce substantial changes in the training of next generations of health professionals and academic teachers.

Keywords: Evaluation of educational programs; Distance education; Covid-19

\footnotetext{
1. Universidade de São Paulo, Faculdade de Medicina de Ribeirão Preto, Departamento de Ginecologia e Obstetrícia, Ribeirão Preto, SP, Brasil 2. Universidade de São Paulo, Faculdade de Medicina de Ribeirão Preto, Departamento de Ciências da Saúde, Ribeirão Preto, SP, Brasil
} 


\section{MENSAGENS PRINCIPAIS}

- A COVID-19 impôs a adoção do ensino remoto emergencial para diversas atividades dos programas de ensino na área da saúde. As mudanças realizadas necessitam de avaliação minimamente fundamentada;

- A função primordial da avaliação de um programa educacional é promover a mudança e a busca constante pela excelência;

- É importante conhecer os princípios de avaliação de programa para entender sua função e aplicações no contexto atual;

- Receber o resultado da avaliação de uma atividade educacional é uma habilidade que deve ser aprendida para que se obtenha o efeito desejado do processo.

\section{INTRODUÇÃO}

A disseminação da infecção pelo novo coronavírus (SARS-CoV-2), também denominada COVID-19, iniciada na China em dezembro de 2019 (1), foi declarada como pandemia pela Organização Mundial de Saúde (OMS) em 11 de março de 2020 (2). O distanciamento social tornou-se rapidamente uma das principais políticas públicas adotadas para reduzir a propagação do vírus e preservar os sistemas de saúde. Essas medidas têm se mostrado eficazes para reduzir a velocidade da disseminação do Covid-19 (3), mas por outro lado, trazem grande impacto para o funcionamento de várias atividades, incluindo as instituições de ensino.

A adoção rápida do ensino remoto emergencial (ERE) tem sido fundamental para a manutenção das atividades educacionais. A educação a distância (EaD) e o ERE tem um grande potencial, ainda pouco explorado e compreendido pelos docentes que, nesta condição adversa tornou-se evidente. Para que esta oportunidade de desenvolvimento seja aproveitada é fundamental que haja reflexão sobre o que tem sido feito e busca contínua pelo aperfeiçoamento nos seus diversos domínios. Neste contexto ganha grande importância a avaliação de programas educacionais.

Vale a pena diferenciar avaliação do estudante, da avaliação dos programas educacionais, que na língua inglesa é mais simples pois existe uma palavra para cada um dos significados, assessment (avaliar estudantes) e evaluation (avaliar qualidade de programas educacionais. Este artigo trata basicamente da avaliação dos programas e que tem por objetivo principal a melhoria contínua e a busca pela excelência. Deste modo, a avaliação de programas educacionais busca reunir dados que possibilitem a revisão e melhoria de todos os processos envolvidos na disciplina ou no curso como um todo. Os cursos de graduação na área de saúde envolvem diversas atividades que visam a formação de um profissional competente. Esta competência envolve diversos domínios que integram conhecimentos, habilidades e atitudes. O programa educacional precisa, portanto, possibilitar este processo de aquisição de competências de forma ampla e equilibrada.

O processo avaliativo deve ser sistemático, contínuo e usado para embasar as ações de aperfeiçoamento. Assim, precisamos entender se o programa está sendo efetivo para atingir os objetivos inicialmente propostos e se os estudantes estão sendo capacitados conforme se pretendia. Um processo de avaliação sólido possibilita que os educadores obtenham informações sobre o programa e sustentem o seu desenvolvimento contínuo (4). Estes conceitos são válidos mesmo em situações de necessidade de rápidas mudanças como estas que estamos vivenciando atualmente.

A avaliação de um programa educacional deve estar necessariamente alinhada com os seus objetivos educacionais, estratégias de ensino, aprendizagem e avaliação do estudante, além de analisar elementos que envolvem corpo docente e técnico administrativo, infraestrutura e recursos disponíveis para sua execução.

É comum encontrarmos referência a avaliação interna e avaliação externa no contexto de avaliação de programas educacionais. Os programas na área da saúde são objeto de avaliação periódica por órgãos legais visando seu credenciamento para continuar suas atividades, por ins- 
tituições que visam fornecer "selos" de acreditação. Este processo é conhecido como avaliação externa e tende a ser realizado em intervalos variáveis. Nos casos dos cursos de graduação da Universidade de São Paulo, por exemplo, a cada cinco anos (ou a depender do resultado da avaliação anterior) ocorre a avaliação pelo Conselho Estadual de Educação para a autorização do recredenciamento dos cursos.

No entanto, quando se considera o objetivo primordial do processo de avaliação, que é a melhoria contínua do programa, talvez as ações mais poderosas sejam aquelas que ocorrem no interior da própria instituição e que constituirão o objeto principal da reflexão feita neste artigo. A avaliação interna deve sempre preceder a avaliação externa. Idealmente este deve ser um processo contínuo, amplo e associado a ações baseadas nos resultados encontrados. Em situações atípicas como a pandemia atual, a utilização dos métodos internos de avaliação das atividades educacionais ganha importância adicional. Avaliar internamente um programa educacional é desafiador, e talvez mais desafiador ainda seja implementar as ações identificadas como necessárias para a melhoria do programa.

\section{CONCEITOS FUNDAMENTAIS PARA A AVALIAÇÃO DE PROGRAMAS EDU- CACIONAIS}

Nós consideramos para efeito de discussão neste artigo o conceito amplo de "programa" como envolvendo desde uma aula única, uma disciplina, um módulo, um estágio ou mesmo um curso como um todo (5). Os conceitos que iremos explorar se aplicam a todas as atividades do processo educacional. As atividades educacionais são de natureza dinâmica, portanto, a maioria dos modelos de avaliação dessas atividades possui como foco as mudanças. A utilização de um referencial teórico é importante para que as estratégias empregadas permitam ir, passo a passo, superando os desafios. Existem diversos modelos que podem ser utilizados para embasar o processo de avaliação de um programa educacional e mesmo de uma determinada disciplina. Neste artigo discutiremos o modelo experimental, o mo- delo de Kirkpatrick, o modelo lógico, e o modelo CIPP (Contexto, Insumo, Processo e Produto).

\section{MODELO EXPERIMENTAL}

O conjunto de desenhos de avaliação que fazem parte do modelo experimental ou quase -experimental segue os preceitos básicos da pesquisa em ciências biomédicas. Tem como pressuposto fundamental a existência de relação linear entre os diversos componentes do programa e os resultados obtidos. O avaliador compara os resultados do programa a partir da análise de grupos submetidos ou não à intervenção. Na forma ideal do modelo experimental, as mudanças no programa seriam avaliadas com a separação de grupos de estudantes de forma randomizada para receberem ou não a intervenção proposta. Após a execução do programa, os resultados dos grupos seriam comparados e a decisão sobre a efetividade da intervenção seria tomada. Esta abordagem já foi tentada em algumas situações, mas sua implementação apresenta inúmeras limitações de natureza prática. Em situações ideais, até seria possível tal desenho aplicado a EaD e ERE, mas na vigência da COVID-19, não é viável. Já os formatos quase-experimentais obedecem à mesma lógica mas assumem desenhos diversos e mais comumente encontrados, tais como ocorrência de cursos paralelos em uma mesma instituição sem alocação aleatória (6), avaliação antes e depois da intervenção comparando grupos distintos em séries temporais (7), ou em um mesmo grupo pela comparação do desempenho antes e depois da intervenção. Desenhos como avaliação de desempenho dos estudantes antes e depois das intervenções educacionais mediadas por tecnologia são viáveis para aplicação no contexto atual e podem fornecer informações importantes para mudanças visando aperfeiçoar as atividades e maximizar os resultados educacionais.

\section{MODELO DE KIRKPATRICK}

O modelo de Kirkpatrick, publicado no ano de 1976, vem sendo aperfeiçoado e adaptado ao longo do tempo. $O$ modelo tem quatro etapas ou 
quatro níveis fundamentais. Baseia-se no preceito de que o estudante satisfeito com as atividades educacionais aprende mais, melhor, busca a aplicação do conhecimento nas suas atividades práticas e, assim, produz efeitos positivos no meio em que está inserido. O modelo recomenda a coleta de dados para avaliar quatro níveis hierárquicos de resultados do programa: satisfação do estudante ou reação ao programa; medidas de aprendizagem atribuídas ao programa (por exemplo, conhecimento adquirido, habilidades aprimoradas, atitudes alteradas); mudanças no comportamento do estudante no contexto para o qual ele está sendo treinado; e os resultados finais do programa em seu contexto mais amplo, como no sistema de saúde (8). Este modelo é de aplicação imediata para as atividades de $E R E$, principalmente nos dois primeiros níveis, que avaliam a satisfação dos estudantes com as atividades e a aprendizagem de conceitos e habilidades. É de grande utilidade para identificar pontos que possam ser corrigidos de uma turma para outra de uma mesma disciplina ou mesmo para duas versões de uma mesma aula.

\section{MODELO LÓGICO}

O modelo lógico busca uma visão mais ampla do programa educacional. Neste modelo, o planejamento e a avaliação do programa ocorrem de forma integrada, o que pode possibilitar ao educador uma visão mais completa do programa. Em geral este modelo é apresentado de forma gráfica e organizado de forma a relacionar os recursos para a implantação das intervenções, as atividades propostas, os resultados das atividades e os impactos educacionais obtidos. O nível de complexidade que se utiliza para avaliar cada um dos componentes do programa é dependente fundamentalmente do objetivo do processo de modificação pretendido e pode variar desde estruturas simples para uma disciplina até processos muito complexos associados a modificações curriculares amplas (9). Este modelo não é de aplicação imediata para as atividades de ERE. No entanto, deve ser considerado no planejamento, fundamentalmente quando se considera a incorporação a médio e longo prazo de atividades edu- cacionais a distância que estão sendo bem sucedidas no momento atual.

\section{MODELO CIPP - CONTEXTO, INSUMO, PROCESSO E PRODUTO}

O modelo CIPP foi proposto a partir da experiência de avaliação do sistema público de ensino elementar e secundário das escolas-distritos de Columbus, Ohio, nos Estados Unidos (10). A avaliação dos quatro componentes, contexto, insumo, processo e produto, utiliza uma diversidade de recursos. O contexto pode ser avaliado pela revisão de documentos, análise demográfica, entrevistas, questionários, grupos focais, análise de registros como desempenho de estudantes em provas. A avaliação dos insumos visando a alocação de recursos geralmente tem como base a revisão de literatura, a visita a programas modelos, avaliação de especialistas e abertura para propostas para interessados em solucionar necessidades identificadas. A avaliação de processo é geralmente utilizada para avaliar a implementação do programa através da observação, revisão documental e entrevistas com participantes do processo. E finalmente a avaliação de produto é similar a avaliação dos resultados dos modelos anteriores e pode usar a avaliação dos usuários, comparação com resultados de programas similares, avaliação da execução dos objetivos propostos, entrevistas com grupos sobre os resultados amplos do programa, estudos selecionados de casos sobre a experiência de participantes do programa, questionários ou depoimentos de participantes sobre os efeitos do programa. Este modelo busca uma visão ampla do programa, requer um engajamento de pessoal e recursos importantes. Apresenta aplicabilidade estratégica para avanços substanciais em programas no seu conceito mais amplo (Ex. curso de graduação, programa de pós-graduação). Pela sua característica, sua aplicação bem sucedida requer engajamento da instituição e tende a ser inviável em iniciativas individuais. Quando pensamos na educação mediada por tecnologia como indutora de mudanças amplas nos cursos na área da saúde, com incorporação novas formas de atenção à saúde das pessoas, 
a utilização deste modelo como forma de avaliação e planejamento das mudanças nos parece de grande potencial. Na Tabela 1 estão resumidos os níveis de aplicação e o potencial de mudança associados aos modelos de avaliação discutidos nesta revisão.

Tabela 1. Modelos de avaliação e aplicabilidade no contexto da educação à distância e COVID-19

\begin{tabular}{lll}
\hline Modelo de avaliação & Nível de Aplicação & Indução de mudança \\
\hline Experimental & Aula, disciplina, curso & Pequenas mudanças \\
Kirkpatrick & Aula, disciplina, curso & Moderadas mudanças \\
Lógico & Disciplina, curso & Moderadas mudanças \\
CIPP & Curso & Grandes mudanças \\
\hline
\end{tabular}

\section{PRINCÍPIOS NORTEADORES PARA O AVALIADOR}

Com o propósito de guiar o trabalho dos profissionais de avaliação e assegurar a ética de sua atuação, a American Evaluation Association (AEA) estabeleceu cinco princípios norteadores aqui resumidos (11):

1. Indagação Sistemática, no que se refere à capacidade de coletar dados utilizando técnicas apropriadas e comunicando métodos e abordagens com a devida transparência para permitir acesso e crítica;

2. Competência, no que se refere a demonstrar atuação competente perante os envolvidos no processo avaliativo e desenvolver continuamente sua capacidade para alcançar o mais alto nível de desempenho possível;

3. Integridade/Honestidade, no que se refere a assegurar honestidade e integridade ao longo de todo o processo avaliativo, negociando com os envolvidos e interessados na avaliação e buscando esclarecer e orientar procedimentos que venham provocar distorções ou indevidas utilizações;

4. Respeito pelas pessoas, no que se refere ao respeito pela segurança, dignidade e auto valorização dos envolvidos no processo avaliativo, atuando sempre com ética profissional, evitando riscos e prejuízos que possam afetar os participantes para assegurar, o melhor possível, o respeito às diferenças e o direito social de retorno dos resultados, aos envolvidos;

5. Responsabilidade pelo bem-estar geral e público, no que se refere a levar em con- sideração a diversidade de interesses e valores que possam estar relacionados ao público em geral, buscando responder não somente às expectativas mais imediatas, mas também às implicações e repercussões mais amplas e, nesse sentido, disseminar a informação sempre que necessário.

\section{COMO COMUNICAR E RECEBER OS RESULTADOS DA AVALIAÇÃO}

Talvez um dos pontos mais desafiadores de um processo de avaliação seja comunicar e receber os resultados do que foi feito. É muito difícil trabalhar efetivamente com a ideia de que a avaliação é um processo voltado para a meIhoria e não para provar a competência das pessoas. Na cultura geral, o resultado da avaliação é frequentemente visto de forma equivocada. Há dois extremos comuns: os resultados da avaliação de atividades que ficam abaixo das expectativas muitas vezes são rejeitados pelos atores que, ao invés de analisá-los em profundidade, concentram-se em questionar sua legitimidade e validade, e os resultados acima das expectativas que, eventualmente, limitam a visão dos atores quanto à possibilidade de mudanças. Mesmo em situações positivas sempre há espaço para meIhoria e progressão. Os resultados de todos os processos de avaliação são sempre oportunidades para se progredir.

O resultado da avaliação, para quem o recebe, apresenta importante similaridade com o feedback para estudantes no processo formativo. $\mathrm{O}$ resultado da avaliação é uma oportunidade ímpar 
para melhorar o programa ou atividade educacional e se aplicam os mesmos princípios para quem recebe a devolutiva de uma avaliação. Algumas características são fundamentais: acreditar que o resultado recebido é uma oportunidade para aperfeiçoamento da atividade educacional, analisar as informações elencadas com atenção antes de responder, quando ficar em dúvida, buscar o esclarecimento, pensar positivamente e manter a abertura para sugestões, aprender com os equívocos e manter a motivação, agir com proatividade e ser grato por ter sua atividade avaliada (12).

\section{CONCLUSÕES}

Conhecer os conceitos de avaliação de programas educacionais é fundamental para a prática docente no momento que atravessamos. A avaliação das atividades educacionais desenvolvidas durante este período pode fornecer valiosas informações para o aperfeiçoamento de aulas, disciplinas e dos cursos no contexto da educação remota e para favorecer mudanças mais profundas a médio e longo prazos.

\section{REFERÊNCIAS}

1. Zhou F, Yu T, Du R, Fan G, Liu Y, Liu Z, et al. Clinical course and risk factors for mortality of adult inpatients with COVID-19 in Wuhan, China: a retrospective cohort study. Lancet Lond Engl. 2020 28;395(10229):1054-62.
2. Bedford J, Enria D, Giesecke J, Heymann DL, Ihekweazu C, Kobinger G, et al. COVID-19: towards controlling of a pandemic. Lancet Lond Engl. 2020 28;395(10229):1015-8

3. Koo JR, Cook AR, Park M, Sun Y, Sun H, Lim JT, et al. Interventions to mitigate early spread of SARS-CoV-2 in Singapore: a modelling study. Lancet Infect Dis. 2020 Jun $1 ; 20(6): 678-88$

4. Goldie J. AMEE Education Guide no. 29: Evaluating educational programmes. Med Teach. 2006 Jan $1 ; 28(3): 210-24$.

5. Frye AW, Hemmer PA. Program evaluation models and related theories: AMEE guide no. 67. Med Teach. 2012;34(5):e288-299.

6. Tavano PT, Almeida MI. Curso Experimental de Medicina na FMUSP e suas conjecturas de implementação. Khronos. $2017 ;(4): 84-101$.

7. Troncon LE de A, Figueiredo JFC, Rodrigues M de LV, Piccinato CE, Peres LC, Cianflone ARL, et al. Avaliação de uma Reestruturação Curricular na Faculdade de Medicina de Ribeirão Preto: Influência sobre o Desempenho dos Graduandos. Rev Bras Educ Médica. 2004 Aug;28(2):145-55

8. Kirkpatrick D. Great Ideas Revisited. Techniques for Evaluating Training Programs. Revisiting Kirkpatrick's Four-Level Model. Train Dev. 1996;50(1):54-9.

9. Frechtling JA. Logic Modeling Methods in Program Evaluation. $1^{a}$ edição. San Francisco: Jossey-Bass; 2007.

10. Stufflebeam DL, Shinkfield AJ. Evaluation Theory Models and Application. 1st edition. San Francisco, Calif.: Jossey-Bass; 2007. 768 p.

11. Guiding Principles for Evaluators [Internet]. [cited 2021 Feb 16]. Available from: https://www.eval.org/About/ Guiding-Principles

12. Hardavella G, Aamli-Gaagnat A, Saad N, Rousalova I, Sreter KB. How to give and receive feedback effectively. Breathe. 2017 Dec;13(4):327-33. 
Corresponding Author:

Anderson Marliere Navarro

navarro@fmrp.usp.br]

Editor:

Prof. Dr. Marcelo Riberto

Recebido: 30/04/2021

Aprovado: $13 / 07 / 2021$

(c) (i) Este é um artigo publicado em acesso aberto (Open Access) sob a licença Creative

sem restrições, desde que o trabalho original seja corretamente citado. 